\title{
Comparison of matched sibling donors versus unrelated donors in allogeneic stem cell transplantation for primary refractory acute myeloid leukemia: a study on behalf of the Acute Leukemia Working Party of the EBMT
}

Eolia Brissot ${ }^{1 *}$ D , Myriam Labopin ${ }^{1,2}$, Matthias Stelljes ${ }^{3}$, Gerhard Ehninger $^{4}$, Rainer Schwerdtfeger ${ }^{5}$, Jürgen Finke ${ }^{6}$, Hans-Jochem Kolb ${ }^{7}$, Arnold Ganser ${ }^{8}$, Kerstin Schäfer-Eckart ${ }^{9}$, Axel R. Zander ${ }^{10}$, Donald Bunjes ${ }^{11}$, Stephan Mielke ${ }^{12}$, Wolfgang A. Bethge ${ }^{13}$, Noël Milpied ${ }^{14}$, Peter Kalhs ${ }^{15}$, Igor-Woflgang Blau ${ }^{16}$, Nicolaus Kröger ${ }^{17}$, Antonin Vitek ${ }^{18}$, Martin Gramatzki ${ }^{19}$, Ernst Holler ${ }^{20}$, Christoph Schmid ${ }^{21}$, Jordi Esteve ${ }^{22}$, Mohamad Mohty ${ }^{1,2}$ and Arnon Nagler ${ }^{23,2}$

\begin{abstract}
Background: Primary refractory acute myeloid leukemia (PRF-AML) is associated with a dismal prognosis. Allogeneic stem cell transplantation (HSCT) in active disease is an alternative therapeutic strategy. The increased availability of unrelated donors together with the significant reduction in transplant-related mortality in recent years have opened the possibility for transplantation to a larger number of patients with PRF-AML. Moreover, transplant from unrelated donors may be associated with stronger graft-mediated anti-leukemic effect in comparison to transplantations from HLA-matched sibling donor, which may be of importance in the setting of PRF-AML.
\end{abstract}

Methods: The current study aimed to address the issue of HSCT for PRF-AML and to compare the outcomes of HSCT from matched sibling donors $(n=660)$ versus unrelated donors $(n=381)$, for patients with PRF-AML between 2000 and 2013. The Kaplan-Meier estimator, the cumulative incidence function, and Cox proportional hazards regression models were used where appropriate.

Results: HSCT provide patients with PRF-AML a 2-year leukemia-free survival and overall survival of about 25 and 30\%, respectively. In multivariate analysis, two predictive factors, cytogenetics and time from diagnosis to transplant, were associated with lower leukemia-free survival, whereas Karnofsky performance status at transplant $\geq 90 \%$ was associated with better leukemia-free survival (LFS). Concerning relapse incidence, cytogenetics and time from diagnosis to transplant were associated with increased relapse. Reduced intensity conditioning regimen was the only factor associated with lower non-relapse mortality.

(Continued on next page)

\footnotetext{
* Correspondence: eolia.brissot@gmail.com

'Service d'Hématologie Clinique et de Thérapie Cellulaire, Hôpital Saint Antoine, APHP, 184 rue du faubourg Saint-Antoine, 75571 Paris, Cedex 12, France

Full list of author information is available at the end of the article
} 
(Continued from previous page)

Conclusions: HSCT was able to rescue about one quarter of the patients with PRF-AML. The donor type did not have any impact on PRF patients' outcomes. In contrast, time to transplant was a major prognostic factor for LFS. For patients with PRF-AML who do not have a matched sibling donor, HSCT from an unrelated donor is a suitable option, and therefore, initiation of an early search for allocating a suitable donor is indicated.

Keywords: Acute myeloid leukemia, Refractory, Allogeneic stem cell transplantation, HLA-matched related donor, Unrelated donor, Graft-versus-host disease

\section{Background}

Primary refractory acute myeloid leukemia (PRF-AML) remains a major therapeutic challenge. There is no agreed definition of PRF-AML, it has been defined by the absence of complete remission (CR), manifested by blast count of $\geq 5 \%$ in bone marrow after one or two cycles of intense induction therapy [1-3]. Recently, less than a $50 \%$ reduction in blast numbers with $>15 \%$ residual blasts after one cycle of induction chemotherapy permits the early identification of patients whose outcome is very poor [4]. CR is achieved in $10-40 \%$ of the cases depending on the AML patient population. Allogeneic stem cell transplantation (HSCT) is the only salvage option with true curative potential in this scenario [5-8]. In the past decade, the use of reduced intensity conditioning regimen (RIC) has significantly increased the proportion of patients with PRF-AML who are eligible for transplant. Indeed, it has been demonstrated that HSCT from matched sibling donors (MSD) is a valid option, leading to disease-free survival rates that are in the range of $20-30 \%$ in these very high risk devastated patient population [7, 9-11]. More recently, PRF-AML were offered HSCT from unrelated donors for (UD), with an overall survival rate (OS) in the range of $22 \%$ [12, 13]. Moreover, HLA mismatch and minor histocompatibility antigen mismatch, which is assumed to be present in fully matched unrelated and also in fully matched related donors, augment donor/recipient alloreactivity, and it has been postulated that histo-incompatibility between unrelated donors and their recipients may be associated with augmented graft-versus-leukemia effect (GVL) and hence lower risks of relapse [14]. However, the various studies comparing the outcomes of AML patients with MSD or UD yielded controversial results, some reporting inferior survival or disease-free survival with UD HSCT [15-18], while others concluded to similar survival rates $[19,20]$. One of the largest AML studies reported similar survival times with MSD and matched UD [21]. Notably, only small series are available concerning PRF-AML. Advances in RIC have significantly increased the proportion of patients with refractory AML who are eligible for transplant. Craddock et al. reported 36 patients who received transplant with a RIC with an UD with an OS at $36 \%$ at 5 years similar to 18 patients with myeloablative conditioning regimen (MAC) [12]. In the past decade, it has been demonstrated that improvement of outcomes where related to the reduction of non-relapse mortality (NRM), majority due to lower infections, lower organ damages, and reduction of severe acute graft-versushost disease (aGVHD) [22]. Because the success of UD HSCT is significantly influenced by the degree of highresolution HLA matching [23] and because advances in supportive care influence outcomes [22, 24, 25], a safety and efficacy comparison of UD versus MSD HSCT in a recent and large cohort of patients is highly warranted to further document decision-making. Therefore, present study based on the European Society of Blood and Marrow Transplantation (EBMT)-Acute Leukemia Working Party (ALWP) database in order to compare outcomes after MSD HSCT versus $10 / 10$ or $9 / 10$ HLA-matched UD HSCT for PRF-AML.

\section{Methods}

\section{Study design and data retrieval}

This is a retrospective, multicenter, registry-based analysis. Data for this study were provided and approved by the Acute Leukemia Working Party of the EBMT group registry. The EBMT registry is a voluntary working group of more than 600 transplant centers, mostly located in Europe, that are required to report all consecutive stem-cell transplantations and follow-up data once a year. Data are entered, managed, and maintained in a central database with internet access; each EBMT center is represented in this database. There are no restrictions on centers for reporting data, except for those required by the law on patient consent, data confidentiality, and accuracy. Quality control measures included several independent systems: confirmation of validity of the entered data by the reporting team, selective comparison of the survey data with MED-A data sets in the EBMT registry database, cross-checking with the National Registries, and regular in-house and external data audits. Since 1990, patients provide informed consent authorizing the use of their personal information for research purposes. 
Eligibility criteria for this analysis included adult patients (aged $>18$ years) with PRF-AML in active disease who had received a first HSCT from HLA-identical sibling or an unrelated donor $(9 / 10$ or $10 / 10)$ with bone marrow or granulocyte colony-stimulating factor-mobilized peripheral blood stem cells. PRF-AML was defined by the failure of achieving $\mathrm{CR}$ (bone marrow blasts $\leq 5 \%$ ) despite induction chemotherapy. All unrelated donors were HLA-matched $(10 / 10)$ or mismatched at one loci (9/10) (-A, -B, -C, -DRB1, -DQB1). We excluded patients who had undergone haploidentical or umbilical cord blood HSCT so that the analysis was restricted to a more homogeneous study population. MAC was defined as per EBMT definition a regimen containing total body irradiation with a dose $>6$ Gy, a total dose of oral busulfan (BU) $>8 \mathrm{mg} / \mathrm{kg}$, or a total dose of intravenous $\mathrm{BU}>6.4 \mathrm{mg} / \mathrm{kg}$ [26]. The sequential strategy consisted of pre-transplant chemotherapy followed by a conditioning regimen [27]. All patients provided informed consent for transplants according to the declaration of Helsinki [26].

\section{End points}

OS was calculated from the date of transplant until death or last observation alive. Leukemia-free survival (LFS) was calculated from the date of transplant until relapse or last disease-free follow-up. Relapse and death from any cause were considered events. NRM was defined as death without prior relapse. Neutrophil recovery was defined as achieving absolute neutrophil count greater than-or equal to- $0.5 \times 109 / 1$ for three consecutive days. The diagnosis and grading of acute [28] and chronic graft-versus-host disease [29] were performed by transplant centers using the standard criteria. Cytogenetics abnormalities were classified according to the European Leukemia Net cytogenetic classification system [30].

\section{Statistical analysis}

Patient-, disease-, and transplant-related variables were compared between the two groups (MSD or UD) using the chi-square statistic for categorical variables and the Mann-Whitney test for continuous variables. Variables considered were patient's age at transplantation, donor/ recipient sex, interval from diagnosis to transplantation, cytogenetics group, type of conditioning (RIC/MAC/ FLAMSA), source of stem cells (peripheral blood stem cell (PBSC) versus bone marrow (BM)), patient/donor CMV serology, Karnofsky performance status (KPS) at time of transplantation, in vivo $\mathrm{T}$ cell depletion, and year of transplantation. Factors that differ significantly between the two groups with $p$ values of $<0.05$ and all factors known as potential prognostic factors were included in the final models. Cumulative incidence functions (CIF) were used to estimate RI and NRM in a competing risk setting, because death and relapse compete with each other. To study chronic graft-versus-host disease (cGVHD), we considered relapse and death to be competing events. Probabilities of LFS and OS were calculated using the Kaplan-Meier estimates. Univariate analyses were performed using Gray's test for CIF, and the log-rank test for LFS and OS. Associations of patient and graft characteristics with outcomes were evaluated in multivariate analysis, using Cox proportional hazards model. All tests were two-sided. The type-1 error rate was fixed at 0.05 for determination of factors associated with time to event outcomes. Statistical analyses were performed with SPSS 22 (SPSS Inc./IBM, Armonk, NY) and R 3.2.2 (R Development Core Team, Vienna, Austria) software packages.

To allow for potential confounding factors between treatments that could influence outcome, propensity score matching was also performed, using the nearest neighbor matching and exact matching for patient age and cytogenetics group. The following factors were included in the propensity score model: patient age (less or more than 50 years), year of transplant, cytogenetics group, patient and donor CMV serology, female donor to male recipient vs other combination, time from diagnosis to transplant, Karnofsky performance status less or more than $90 \%$ at $\mathrm{HSCT}$, and use of in vivo T cell depletion and conditioning (MAC/RIC/FLAMSA). Owing to the significant differences in baseline characteristics between the MSD and UD groups, caliper matching was fixed to 0.2 . The purpose of the propensity score matching strategy was to reduce confounding effects of these variables and strengthen causal inferences. Propensity score analysis was performed using the "MatchIt" (Ref: Package "MatchIt". 2015 (accessed: 18 May 2015). http://cran.pro ject.org/web/packages/MatchIt/MatchIt.pdf). Comparisons between the two match-paired groups were stratified on matching group for taking into account for association using either mixed effects Cox model.

\section{Results}

Patients, disease and transplant characteristics

We obtained data from 211 reporting centers (Additional file 1), and 104 of whom used both types of donors. Patient and disease characteristics are summarized in Table 1 . Six hundred sixty patients received a MSD and 381 patients an UD (296 with an HLA-matched 10/10, and 85 with a mismatched 9/10). The two patient cohorts were different for several variables (Table 1). The median year of transplant in the MSD group was 2007 (range 2000-2013), whereas patients with UD underwent HSCT more recently (median 2010, range 2000-2013; $p<10^{-5}$ ). Median follow-up for all patients was 39 months (32.547.5) but median follow-up was longer in the MSD group (48 months [range, 40.1-157.2]) than in the UD group (30 months [24.7-36.2], $p=0.04$ ). All patients were refractory in active phase of disease at time of HSCT as per the 
Table 1 Baseline characteristics of patients

\begin{tabular}{|c|c|c|c|}
\hline & MSD & UD & $p$ value \\
\hline Size, $n$ & 660 & 381 & \\
\hline Centers, $n$ & 199 & 104 & \\
\hline $\begin{array}{l}\text { Median follow-up, } \\
\text { months (range) }\end{array}$ & $48(40.1-157)$ & $30(27-36)$ & 0.04 \\
\hline Year of Tx (range) & $2007(2000-13)$ & $2010(2000-13)$ & $<10^{-5}$ \\
\hline $\begin{array}{l}\text { Interval diagnosis to } T x \text {, } \\
\text { day (range) }\end{array}$ & $110(60-180)$ & $111(60-178)$ & 0.33 \\
\hline \multicolumn{4}{|l|}{ Patient sex, n (\%) } \\
\hline Male & $393(59.5)$ & $212(55.8)$ & \multirow[t]{2}{*}{0.24} \\
\hline Female & $267(40.5)$ & $168(44.2)$ & \\
\hline \multicolumn{4}{|l|}{ Donor sex, $n(\%)$} \\
\hline Male & $347(53)$ & $253(71)$ & \multirow[t]{2}{*}{$<10^{-5}$} \\
\hline Female & $308(47)$ & $103(29)$ & \\
\hline \multicolumn{3}{|l|}{ Cytogenetics, $n(\%)$} & \multirow[t]{5}{*}{0.02} \\
\hline Good & $13(4.9)$ & $4(2.9)$ & \\
\hline intermediate & $170(64.2)$ & $71(51.8)$ & \\
\hline Poor & $82(30.9)$ & $62(45.3)$ & \\
\hline Unknown/failed & 395 & 244 & \\
\hline \multicolumn{4}{|l|}{ KPS, $n(\%)$} \\
\hline$<90 \%$ & $183(29.8 \%)$ & $119(33 \%)$ & \multirow[t]{2}{*}{0.30} \\
\hline$\geq 90 \%$ & $431(70.2 \%)$ & $242(67 \%)$ & \\
\hline \multicolumn{4}{|c|}{ Female $\mathrm{D}$ to male $\mathrm{R}, n(\%)$} \\
\hline No & $467(71.3)$ & $306(86.2)$ & \multirow[t]{2}{*}{$<10^{-5}$} \\
\hline Yes & $188(28.7)$ & $49(13.8)$ & \\
\hline \multicolumn{4}{|l|}{ CMV patient, $n(\%)$} \\
\hline Negative & $162(30.5)$ & $148(40)$ & \multirow[t]{2}{*}{0.003} \\
\hline Positive & $369(69.5)$ & $222(60)$ & \\
\hline \multicolumn{4}{|l|}{ CMV donor, $n(\%)$} \\
\hline Negative & $203(38.8)$ & $204(54.4)$ & \multirow[t]{2}{*}{$<10^{-5}$} \\
\hline Positive & $320(61.2)$ & $171(45.6)$ & \\
\hline \multicolumn{4}{|l|}{ Source of stem cell, $n(\%)$} \\
\hline BM & $53(8)$ & 20 & \multirow[t]{2}{*}{0.09} \\
\hline PBSC & $607(92)$ & $361(94.8)$ & \\
\hline \multicolumn{4}{|c|}{ Conditioning regimen, $n$ (\%) } \\
\hline MAC & $373(56.5)$ & $169(44.4)$ & \multirow[t]{3}{*}{$<10^{-4}$} \\
\hline RIC & $193(29.2)$ & $93(24.4)$ & \\
\hline Sequential strategy & $94(14.3)$ & $119(31.2)$ & \\
\hline \multicolumn{4}{|l|}{ In vivo T depletion, $n$ (\%) } \\
\hline No & $357(65.6)$ & $73(19.5)$ & \multirow[t]{2}{*}{$<10^{-5}$} \\
\hline Yes & $187(34.4)$ & $302(80.5)$ & \\
\hline \multicolumn{4}{|l|}{ GVHD prophylaxis, n (\%) } \\
\hline CsA alone & $85(12.9)$ & $44(11.5)$ & \\
\hline CsA+MTX & $256(38.8)$ & $93(24.5)$ & \\
\hline CsA+ MMF + other & $165(25)$ & $194(50.9)$ & \\
\hline
\end{tabular}

Abbreviations: BM bone marrow, CSA cyclosporine, $D$ donor, KPS Karnofsky Performance Status, MAC myeloablative conditioning regimen, $M S D$ matched sibling donor, MMF mycophenolate mophetyl, MTX methotrexate, PBSC peripheral blood stem cell, $R$ recipient, RIC reduced intensity conditioning regimen, $T x$ transplantation, UD unrelated donor, GVHD graft-versus-host disease study inclusion criteria. Median age was higher in the UD group (50.5 years $(18-74)$ vs 47.7 years $(18-74)$ in the MSD group, $p=0.006)$. The median time from diagnosis to HSCT was similar in the two groups (110 days [60-180] vs 111 days [60-178], respectively, $p=0.33$ ). In the MSD group, 56.5\% received a MAC regimen, $29.2 \%$ a RIC regimen, and $14.3 \%$ a sequential conditioning regimen mainly FLAMSA (fludarabine, cytarabine, and amsacrine) followed by conditioning regimen, while in the UD group, $44.4 \%$ received a MAC regimen, $24.4 \%$ a RIC regimen, and $31.2 \%$ a sequential conditioning regimen $\left(p<10^{-4}\right)$. The MSD group contained more cytomegalovirus-positive recipients and recipients with cytomegalovirus-positive donors than did the UD group (Table 1). Cyclosporine and methotrexate were used as the main graft-versus-host disease prophylaxis in the MSD group. The proportion of patients who received in vivo $\mathrm{T}$ cell depletion significantly differed between the two groups $(34.4 \%$ in the MSD group and $80.5 \%$ in the UD group, $p<10^{-5}$ ). PBSC was by far the main stem cell source in both groups $(92 \%$ in the MSD group vs $94.8 \%$ in the UD group, $p=0.09$ ) (Table 1 ).

\section{Engraftment, GvHD, and response}

Cumulative incidence (CI) of neutrophil engraftment was similar with a MSD and UD, 94.2 and $95.2 \%$, respectively $(p=0.5)$. Day of absolute neutrophil count $\geq 500$ cells per $\mu \mathrm{L}$ did not differ as well between HSCT from MSD vs UD, 15 days (6-43) and 15 days (1-43) days after HSCT, respectively $(p=0.76)$ (Table 2$)$.

As expected, lower incidence of all grades aGVHD was observed post-HSCT with a MSD than a UD; indeed, CI of grades II-IV were 27.9 and $35.5 \%$, respectively ( $p=$ 0.012). However, no difference was observed in the rates of severe grade III-IV aGVHD (12.3\% in the MSD group and $16.1 \%$ in the UD group, $(p=0.09)$ (Table 2$)$ ).

At 2 years, the cumulative incidence of cGVHD was similar in the two groups (28.9 and $25.8 \%$, respectively,

Table 2 Transplantation outcomes

\begin{tabular}{llll}
\hline & MSD $(n=660)$ & UD $(n=381)$ & $p$ value \\
\hline Engraftment & $606(94.2 \%)$ & $359(95.2 \%)$ & 0.5 \\
Acute GVHD & & & 0.012 \\
$\quad$ Grade 0-I & $447(72.1 \%)$ & $236(64.5 \%)$ & \\
$\quad$ Grade II-IV & $173(27.9 \%)$ & $130(35.5 \%)$ & \\
Outcome at 2 years & & & \\
$\quad$ Leukemia-free survival & $25.3 \%(21.6-28.9)$ & $28.3 \%(23.3-33.3)$ & 0.56 \\
Overall survival & $30.9 \%(27-34.9)$ & $34.3 \%(29-39.7)$ & 0.57 \\
$\quad$ Relapse & $53.7 \%(49.6-57.7)$ & $46.4 \%(40.9-51.7)$ & 0.04 \\
$\quad$ Non-relapse mortality & $21 \%(17.9-24.4)$ & $25.1 \%(21.7-28.6)$ & 0.11 \\
Chronic GVHD & $28.9 \%(24.9-33)$ & $25.8 \%(20.8-31)$ & 0.77 \\
\hline
\end{tabular}

Data are $n(\%)$ or $n(\% ; 95 \% \mathrm{Cl})$

MSD matched sibling donors, UD unrelated donors, GVHD graft-versus-host disease 
$p=0.56$ ) (Table 2 ). $\mathrm{T}$ cell in vivo depletion mostly represented by ATG was the only favorable factor to prevent cGVHD (HR $=0.51,95 \%$ CI, 0.35-0.74, $p=0.001$ ) (Table 3). When investigating the effect of cGVHD on the incidence of relapse using Cox with time-dependent variables (univariate analysis), we found a significant association between the two events ( $\mathrm{HR}=0.60,95 \% \mathrm{CI}, 0.45-0.81, p=0.001)$.

In terms of response, after MSD and UD, 443 patients (71\%) in the MSD group and 247 (69\%) patients in the UD obtained a CR after HSCT, while 148 (24\%) and 93 (26\%) did not achieved complete remission. Thirty-three (5.3\%) patients in the MSD group and 20 (5.6\%) in the UD group were not evaluable for response because of early death, respectively $(p=0.73)$.

\section{LFS, OS, RI, and NRM}

In univariate analysis, LFS at 2 years was $25.3 \%$ in the MSD group vs $28.3 \%$ in the UD group, respectively $(p=0.56)$ (Fig. 1a). In univariate analysis, in intermediate cytogenetic group, LFS between the two types of donors MSD and UD was 29.5\% (22.5-36.5) and 35.7\% (24.2$47.2)(p=0.34)$, respectively In the group with poor cytogenetic, LFS at 2 years was $16 \%(7.5-24.4)$ in patients receiving a MSD and 19.8\% (9.4-30.1) in patients with an UD $(p=0.37)$; OS at 2 years was $21 \%(11.4-30.7)$ in the MSD group and 25.2\% (13.9-36.5) in the UD group $(p=0.89)$. Multivariate analysis showed lower LFS in patients who had a poor cytogenetics compared to those with intermediary risk $(\mathrm{HR}=1.61,95 \% \mathrm{CI}, 1.24-2.09$, $p=0.0004)$ and when transplant was performed above the median of 110 days from diagnosis ( $\mathrm{HR}=1.21,95 \% \mathrm{CI}$, $1.02-1.44, p=0.03$ ), whereas KPS at transplant $\geq 90 \%$ was associated with better LFS (HR $=0.67,95 \%$ CI, 0.56-0.80, $p=0.0001$ ). We noted no effect for donor types, year of transplant, patient or donor cytomegalovirus (CMV) status, in vivo $\mathrm{T}$ depletion, and type of conditioning regimen (Table 3).

OS at 2 years did not differ between the two groups (30.9\% in the MSD group vs $34.3 \%$ in the UD group $(p=0.57))$ (Fig. 1b). In univariate analysis, in the intermediate cytogenetic group, OS did not differ statistically between the two types of donors MSD and UD (37.1\% (29.6-44.5) and 42.6\% (30.7-54.4) respectively, $p=0.226)$. In the group with poor cytogenetic, OS at 2 years was $21 \%(11.4-30.7)$ in the MSD group and $25.2 \%(13.9-36.5)$ in the UD group $(p=0.89)$. OS at 2 years did not statistically differ between the group with in vivo $\mathrm{T}$ cell-depleted graft and no $\mathrm{T}$ cell-depleted graft ( $p=0.49$ ) (Additional file 1: Table S1). Looking at the conditioning regimen, no difference was observed between MAC, RIC, and sequential strategy $(p=0.602)$ (Additional file 1: Table S1). These results were confirmed by multivariate analysis. In multivariate analysis, four predictive factors were associated with lower OS: age $>50$ years, cytogenetics (poor vs intermediate), time from diagnosis to transplant, and CMV-positive status, whereas KPS at transplant $\geq 90 \%$ was associated with a better OS (Table 3).

We noted a higher RI in the MSD group compared to the UD group (53.7 and 46.4\%, $p=0.04$; respectively) (Table 2, Fig. 1c). However, in multivariate analysis for RI, only cytogenetics (poor vs intermediate) and time from diagnosis to transplant were the only risk factors associated with increased RI [ $(\mathrm{HR}=1.74,95 \% \mathrm{CI}, 1.30$ 2.33, $p=0.0002)(\mathrm{HR}=1.29,95 \% \mathrm{CI}, 1.06-1.58, p=0.01)$, respectively] whereas KPS at transplant was a protective factor $(\mathrm{HR}=0.77,95 \% \mathrm{CI}, 0.62-0.95, p=0.01)$ (Table 3).

No difference in NRM was noted between the two groups in univariate analysis (Table 2, Fig. 1d). However, multivariate analysis demonstrated that patient age ( $\geq 50$ years), and CMV-positive status were associated with higher NRM $(\mathrm{HR}=1.77,95 \% \mathrm{CI}, 1.27-2.47, p=0.001 ; \mathrm{HR}=1.68$, 95\% CI, 1.14-2.47, $p=0.008$ ), while RIC regimen compared to MAC regimen was the only factor associated with lower NRM (HR $=0.59,95 \% \mathrm{CI}, 0.41-0.85, p=0.005)$ (Table 3). We noted no effect for donor types.

Finally, in order to reduce the effects of confounding factors, we did a propensity score analysis. Using a caliper of 0.2 , we were able to match 221 MSD and 221 UD on the following variables: patient age (less or more than 50 years), year of transplant, cytogenetics group, patient and donor CMV serology, female donor to male recipient versus other combination, time from diagnosis to transplant, Karnofsky performance status less or more than 90\% at HSCT, and use of in vivo T cell depletion and conditioning (MAC/RIC/FLAMSA). The results of the Cox analysis were confirmed in this subpopulation of patients with balanced characteristics as described in Table 4.

\section{Causes of death}

AML was the most common cause of death $(64.9 \%$ in the MSD group and $55.3 \%$ in the USD group). GVHD represented $14.2 \%$ of death causes in the MSD group and $16.1 \%$ in the UD group. Infection was the death cause in 10.8 and $19.4 \%$ for the MSD and UD groups, respectively.

\section{Discussion}

In the present study, we addressed the topic of HSCT for patients with PRF-AML a devastating medical condition with no other therapeutic option for cure. Specifically, we compared the transplantation outcomes after MSD versus UD (10/10 or 9/10). Our cohort included 1041 patients: 660 received MSD HSCT and 381 a UD HSCT (296 10/10 matched UD HSCT, and 85 9/10 matched UD). We were able to show about 25\% LFS and 30\% OS post-HSCT for this high-risk advanced disease with no difference between sibling vs unrelated HSCT. Despite higher rates of grade II-IV aGVHD in the UD group, neither the 2-year cumulative incidence of severe grade 
Table 3 Multivariate analysis for LFS, OS, RI, NRM, and CGVHD

\begin{tabular}{|c|c|c|c|c|}
\hline \multirow{2}{*}{$\overline{\mathrm{LFS}}$} & \multirow[t]{2}{*}{$\mathrm{HR}$} & \multicolumn{2}{|c|}{$95 \% \mathrm{Cl}$} & \multirow{2}{*}{$p$ value } \\
\hline & & & & \\
\hline UD vs MSD & 0.96 & 0.79 & 1.17 & 0.68 \\
\hline Age $\geq 50$ years & 1.17 & 0.98 & 1.40 & 0.09 \\
\hline Year of $T x>$ median & 1.02 & 0.85 & 1.23 & 0.83 \\
\hline \multicolumn{5}{|l|}{ Cytogenetics } \\
\hline Poor vs intermediate & 1.61 & 1.24 & 2.09 & $<10^{-3}$ \\
\hline Missing vs intermediate & 1.18 & 0.96 & 1.46 & 0.11 \\
\hline CMV patient positive & 1.19 & 0.98 & 1.44 & 0.09 \\
\hline CMV donor positive & 0.99 & 0.82 & 1.19 & 0.90 \\
\hline Female to male & 1.03 & 0.84 & 1.26 & 0.77 \\
\hline Diag to $T x>$ median & 1.21 & 1.02 & 1.44 & 0.03 \\
\hline KPS $>90 \%$ & 0.67 & 0.56 & 0.80 & $<10^{-5}$ \\
\hline In vivo T depletion & 1.02 & 0.84 & 1.26 & 0.81 \\
\hline \multicolumn{5}{|l|}{ Conditioning regimen $(r e f=M A C)$} \\
\hline RIC VS MAC & 0.89 & 0.73 & 1.08 & 0.25 \\
\hline Sequential strategy vs MAC & 0.91 & 0.69 & 1.21 & 0.53 \\
\hline \multicolumn{5}{|l|}{ OS } \\
\hline UD vs MSD & 1.02 & 0.83 & 1.25 & 0.87 \\
\hline Age $\geq 50$ years & 1.33 & 1.10 & 1.61 & 0.004 \\
\hline Year of $T x>$ median & 1.02 & 0.84 & 1.24 & 0.83 \\
\hline \multicolumn{5}{|l|}{ Cytogenetics } \\
\hline Poor vs intermediate & 1.55 & 1.19 & 2.03 & 0.001 \\
\hline Missing vs intermediate & 1.23 & 0.99 & 1.53 & 0.06 \\
\hline CMV patient positive & 1.28 & 1.04 & 1.58 & 0.02 \\
\hline CMV donor positive & 0.97 & 0.80 & 1.19 & 0.79 \\
\hline Female to male & 1.06 & 0.86 & 1.32 & 0.57 \\
\hline Diag to $T x>$ median & 1.20 & 1.00 & 1.44 & 0.05 \\
\hline KPS $>90 \%$ & 0.65 & 0.54 & 0.79 & $<10^{-5}$ \\
\hline In vivo $T$ depletion & 0.94 & 0.76 & 1.16 & 0.55 \\
\hline \multicolumn{5}{|l|}{ Conditioning regimen (ref = MAC) } \\
\hline RIC VS MAC & 0.83 & 0.67 & 1.02 & 0.08 \\
\hline Sequential strategy vs MAC & 0.81 & 0.60 & 1.09 & 1.16 \\
\hline \multicolumn{5}{|l|}{$\mathrm{Rl}$} \\
\hline UD vs MSD & 0.89 & 0.71 & 1.12 & 0.33 \\
\hline Age $\geq 50$ years & 0.99 & 0.80 & 1.22 & 0.94 \\
\hline Year of Tx >median & 0.95 & 0.77 & 1.18 & 0.66 \\
\hline \multicolumn{5}{|l|}{ Cytogenetics } \\
\hline Poor vs intermediate & 1.74 & 1.30 & 2.33 & $<10^{-3}$ \\
\hline Missing vs intermediate & 1.10 & 0.86 & 1.40 & 0.45 \\
\hline CMV patient positive & 1.07 & 0.86 & 1.33 & 0.56 \\
\hline CMV donor positive & 0.97 & 0.78 & 1.20 & 0.75 \\
\hline Female to male & 1.08 & 0.86 & 1.37 & 0.51 \\
\hline Diag to $T x>$ median & 1.29 & 1.06 & 1.58 & 0.01 \\
\hline KPS $>90 \%$ & 0.77 & 0.62 & 0.95 & 0.01 \\
\hline
\end{tabular}

Table 3 Multivariate analysis for LFS, OS, RI, NRM, and CGVHD (Continued)

\begin{tabular}{|c|c|c|c|c|}
\hline In vivo $T$ depletion & 1.10 & 0.87 & 1.40 & 0.43 \\
\hline \multicolumn{5}{|l|}{ Conditioning regimen $(r e f=M A C)$} \\
\hline RIC vs MAC & 1.03 & 0.81 & 1.29 & 0.83 \\
\hline Sequential strategy vs MAC & 0.98 & 0.71 & 1.35 & 0.89 \\
\hline \multicolumn{5}{|l|}{ NRM } \\
\hline UD vs MSD & 1.30 & 0.91 & 1.86 & 0.14 \\
\hline Age $\geq 50$ years & 1.77 & 1.27 & 2.47 & 0.001 \\
\hline Year of $\mathrm{Tx}>$ median & 1.18 & 0.84 & 1.64 & 0.33 \\
\hline \multicolumn{5}{|l|}{ Cytogenetics } \\
\hline Poor vs intermediate & 1.53 & 0.91 & 2.59 & 0.11 \\
\hline Missing vs intermediate & 1.44 & 0.98 & 2.13 & 0.06 \\
\hline CMV patient positive & 1.68 & 1.14 & 2.47 & 0.01 \\
\hline CMV donor positive & 1.06 & 0.75 & 1.49 & 0.76 \\
\hline Female to male & 1.01 & 0.70 & 1.47 & 0.95 \\
\hline Diag to $T x>$ median & 1.06 & 0.77 & 1.45 & 0.72 \\
\hline KPS $>90 \%$ & 0.48 & 0.35 & 0.66 & $<10^{-5}$ \\
\hline In vivo T depletion & 0.89 & 0.62 & 1.29 & 0.54 \\
\hline \multicolumn{5}{|c|}{ Conditioning regimen (ref $=\mathrm{MAC}$ ) } \\
\hline RIC vs MAC & 0.59 & 0.41 & 0.85 & 0.005 \\
\hline Sequential strategy vs MAC & 0.82 & 0.48 & 1.38 & 0.45 \\
\hline \multicolumn{5}{|l|}{ cGVHD } \\
\hline UD vs MSD & 1.22 & 0.86 & 1.74 & 0.26 \\
\hline Age $\geq 50$ years & 1.06 & 0.76 & 1.47 & 0.73 \\
\hline Year of Tx > median & 1.06 & 0.76 & 1.48 & 0.72 \\
\hline \multicolumn{5}{|l|}{ Cytogenetics } \\
\hline Poor vs intermediate & 1.01 & 0.64 & 1.59 & 0.96 \\
\hline Missing vs intermediate & 0.82 & 0.58 & 1.16 & 0.26 \\
\hline CMV patient positive & 1.24 & 0.87 & 1.75 & 0.23 \\
\hline CMV donor positive & 1.07 & 0.76 & 1.51 & 0.70 \\
\hline Female to male & 1.53 & 1.07 & 2.19 & 0.02 \\
\hline Diag to $T x>$ median & 0.85 & 0.62 & 1.17 & 0.32 \\
\hline KPS $>90 \%$ & 1.01 & 0.72 & 1.41 & 0.97 \\
\hline In vivo $T$ depletion & 0.51 & 0.35 & 0.74 & 0.001 \\
\hline \multicolumn{5}{|l|}{ Conditioning regimen $(r e f=M A C)$} \\
\hline RIC vs MAC & 1.20 & 0.83 & 1.73 & 0.32 \\
\hline Sequential strategy vs MAC & 1.07 & 0.63 & 1.80 & 0.81 \\
\hline
\end{tabular}

Abbreviations: Cl confidence interval, Diag diagnosis, GVHD graft-vs-host disease, $H R$ hazard ratio, KPS Karnofsky Performance Status, LFS leukemia-free survival, MAC myeloablative conditioning, MSD matched sibling donor, NRM non-relapse mortality, OS overall survival, RIC reduced intensity conditioning, $T X$ transplantation, UD unrelated donor

III-IV aGVHD, nor cGVHD or the 2-year NRM rates differed significantly between the two groups (Tables 2 and 3 ). Although we could hypothesize a stronger graft-versusleukemia effect post UD compared to MSD, we did not observed difference in terms of RI or LFS. Even in the setting 


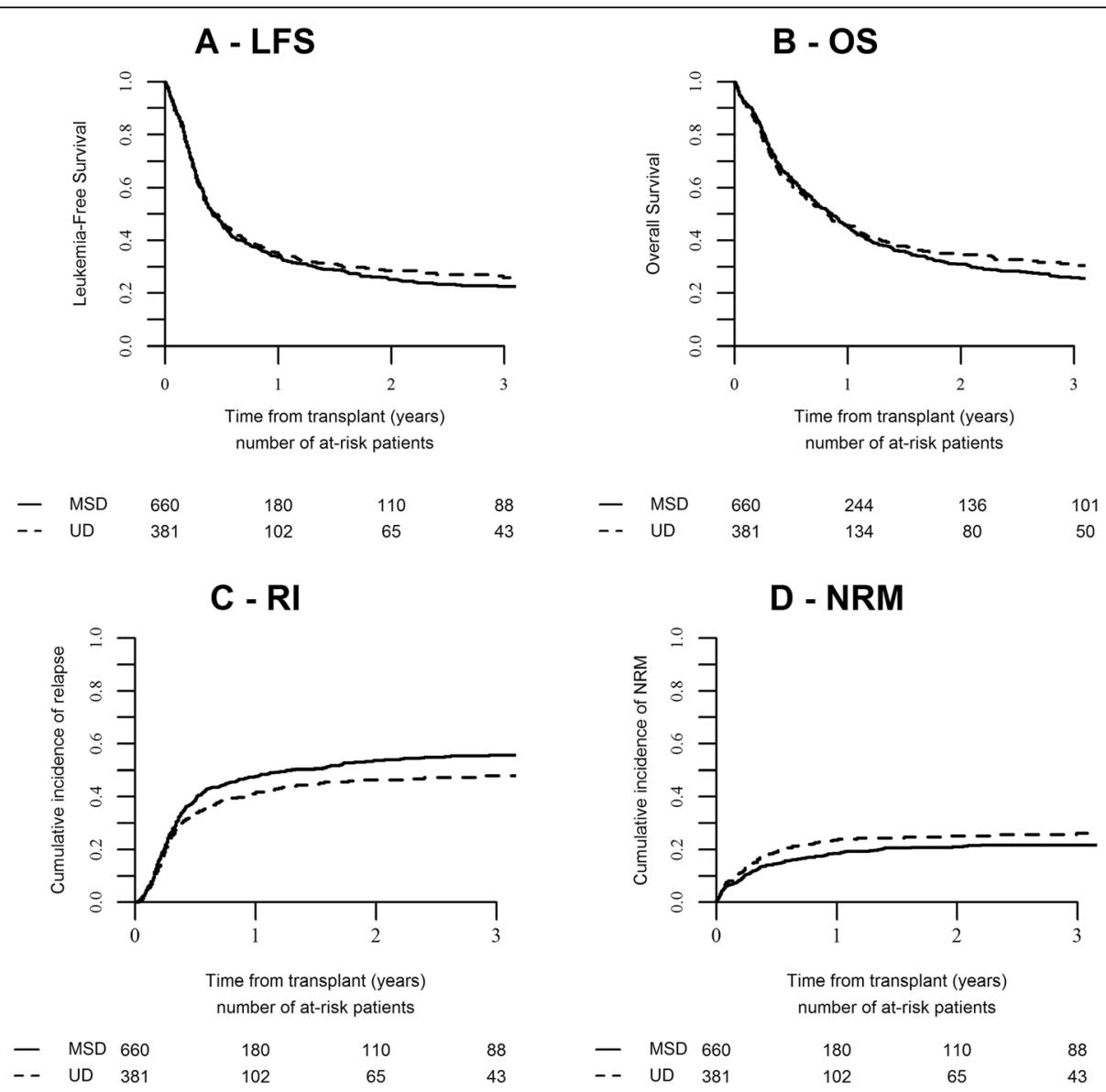

Fig. 1 Probability of a leukemia-free survival (LFS), b overall survival (OS), c relapse incidence (RI), and $\mathbf{d}$ non-relapse mortality (NRM) in allografted patients with PRF-AML

of maximum HLA disparity namely haploidentical transplants, we could not observed lower relapse rate as compared to HLA-matched sibling HSCT [20, 31, 32]. One theoretic explanation is that due to the very aggressive biology of the leukemia being refractory to chemotherapy disease, progression and relapse occur early after transplant as even the MAC or FLAMSA conditioning are unable to control the disease while (i) it takes time (up to 9 months) for the GVL effect to be established and to work against the leukemia and (ii) in order to be effective, a state of

Table 4 Propensity score analysis for LFS, OS, RI, NRM and cGVHD

\begin{tabular}{lllc}
\hline & MSD & UD & $p$ value (frailty) \\
\hline LFS & $25.7 \%(19.1-32.3)$ & $30.1 \%(23.4-36.8)$ & 0.56 \\
OS & $31.3 \%(24.1-38.6)$ & $38.2 \%(31.1-45.4)$ & 0.94 \\
RI & $54.3 \%(46.8-61.1)$ & $45.6 \%(38.3-52.5)$ & 0.11 \\
NRM & $20 \%(14.6-26.1)$ & $24.1 \%(18.4-30.2)$ & 0.19 \\
CGVHD & $24.2 \%(17.8-31.2)$ & $30.3 \%(23.4-37.5)$ & 0.08 \\
\hline
\end{tabular}

Abbreviations: CGVHD chronic graft-vs-host disease, LFS leukemia-free survival, MSD matched sibling donor, NRM non-relapse mortality, OS overall survival, UD unrelated donor minimal residual disease or at least substantial reduction in the leukemic tumoral mass need to be achieved [33, 34].

In our study, 27.5\% of patients received a RIC regimen. As it is PRF, NRM was significantly lower in the RIC group compared to the MAC group, but there were no statistically differences in LFS, OS, and RI. This study had included 213 HSCT patients who received sequential approach with aplasia-inducing chemotherapy followed by conditioning regimen. Schmid et al. found a 2-year OS of $40 \%$ and a 2 -year NRM of $22 \%$ using FLAMSA followed by $\mathrm{TBI} /$ cyclophosphamide in patients with refractory AML who received a MSD or a UD [35]. Stelljes et al. had shown previously that the 2-year OS with RIC in AML patients in complete remission versus untreated or refractory disease from related or unrelated donors is 81 and $21 \%$, respectively $[32,36]$. This underlines the potential effect of prior leukemic burden reduction to improve survival. In the current study, no outcome differences were found between MAC, RIC, or sequential regimens. Due to the aggressive biology of the leukemia and the possible refractoriness to chemotherapy of the malignant leukemic clone, it is conceivable that even the most intense chemotherapy 
conditioning is unable to induce remission or even transient response which allow sufficient time for the alloreactive cells to mediate the GVL effect [34]. Future prospective studies should more specifically address the outcome differences between these approaches.

Our data identified that proceeding to HSCT as soon as possible was one of the most important factors determining the PRF-AML outcome. Indeed, time from diagnosis to transplant longer than the median (110 days) was a negative prognostic factor for LFS, OS, and RI in multivariate analysis. These data are consistent with the study of Craddock et al. [12] and emphasize the urgent need of searching for an UD for PRF-AML patients who lack a matched sibling donor. We may add that this is a factor we can influence as oppose to most of other factors like disease biology, cytogenetics, age, and comorbidities. Thus, we recommend in patients that failed two lines of therapy not to try additional lines to achieve CR but to do a fast search and to proceed to transplant.

Cytogenetic features of the AML represent a major prognostic factor in LFS, RI, and OS [37, 38]. Duval et al. reported that poor-risk cytogenetics was an adverse pre-HSCT variable in patients with PRF-AML or relapsed AML and who underwent a MAC regimen [6]. Thereby, PRF-AML with poor cytogenetic characteristics were associated with statistically significant lower LFS, higher RI, and lower OS at 2 years in multivariate analysis. These data pave the road for investigating more intensive additional approaches relying on sequential conditioning regimens and/or post-transplant treatments such as 5azacytidine, prophylactic donor lymphocytes infusions, or targeted therapy in order to further improve in the prognosis of this devastating subgroup of patients [39].

Several studies have investigated the prognostic impact of KPS at transplant $[6,40,41]$. KPS was independently correlated with toxicities, NRM, and overall mortality [40]. In our study, KPS at HSCT was of prognostic value. Indeed, KPS, when stratified into scores of less than $90 \%$ and greater than $90 \%$ at HSCT, had a significant predictive value for LFS, OS, RI, and NRM. This simple parameter is a powerful predictor of post-HSCT outcomes and may therefore contribute to guide our practical management of PRF-AML.

Being a retrospective study has few limitations: patient characteristics vary among the groups for several factors including year of transplant, age, patient and recipient CMV status, and cytogenetic risk. There is a relative inherent selection process for HSCT in our study, since the patients corresponded to a subgroup who fitted the criteria to undergo HSCT. Also, we do not have information about why patients were allocated to a specific donor in the registry and distinguishing the choice of the donor from the role of a potential center effect is difficult. Finally, number of circulating and bone marrow blasts at time of HSCT are missing in substantial number of patients. However, the aim of this analysis was to compare the two types of donors using EBMT registry data. The design of the study and inclusion criteria were intended to answer this clinical question and therefore are not adapted to develop a prognostic score based on information that is not routinely collected in the registry. Unfortunately, no ongoing trials are comparing outcomes after MSD or UD for PRFAML. Therefore, in the absence of any prospect of such comparative studies, our data suggest that both donors are equally effective in these very high risk patients.

\section{Conclusions}

Our results suggest that, when an HLA-identical sibling donor is not available for an adult with PRF-AML who is otherwise a candidate for HSCT, a 10/10 or 9/10 UD may be used with the expectation of similar rates of NRM, LFS, and OS at 2 years. However, despite this expanded use, as many as one-third of patients will not get a UD donor source, so that alternative options such as haploidentical donors and use of cord blood stem cells may need consideration and evaluation. In these circumstances, we would recommend to rather proceed fast to transplant than multiplying the number of lines of chemotherapy that would increase toxicity without achieving an anti-leukemic response and thus will hamper the chance to reach HSCT that may rescue about $25 \%$ of the patients. Disease relapse remains the most common cause of treatment failure after HSCT for this group of patients with PRF-AML. Potential approaches to reduce this high risk of AML relapse following HSCT could be prophylactic or preemptive therapy. Few prospective single-arm studies investigating hypomethylating agents (5-azacytidine or decitabine) as consolidation therapy for patients with AML or myelodysplastic syndrome after HSCT have been published [42-46]. Given the limited number of patients and the lack of a control arm, a definitive ranking of outcome results is of course difficult so far but they suggested a potential benefit impact. Panobinostat, a potent inhibitor of deacetylases, maintenance post-HSCT was reported to be feasible and associated with a low relapse rate [47]. Sorafenib maintenance has been recently reported in 27 patients with FTL3-ITD+ with encouraging results [48]. In all, these data suggest that primary refractory AML patients should be transplanted as quickly as possible with either a MSD or an UD and maintenance therapy should be a very promising approach for these very high risk patients.

\section{Additional file}

Additional file 1 Table 1S: Conditioning regimens: univariate analysis for RI, NRM, LFS, OS, CGVHD. (DOCX $20 \mathrm{~kb}$ ) 


\section{Abbreviations}

aGVHB: Acute graft-versus-host disease; ALWP: Acute leukemia working party; AML: Acute myeloid leukemia; BM: Bone marrow; BU: Busulfan; CGVHD: Chronic graft-versus-host disease; CIF: Cumulative incidence functions; CMV: Cytomegalovirus; CR: Complete remission; EBMT: European Society of Blood and Marrow Transplantation; FLAMSA: Fludarabine, cytarabine, and amsacrine; GVL: Graft-versus-leukemia effect; HLA: Human leukocyte antigen; HSCT: Allogeneic stem cell transplantation; KPS: Karnofsky performance status; LFS: Leukemia-free survival; MAC: Myeloablative conditioning regimen; MSD: Matched sibling donor; NRM: Non-relapse mortality; OS: Overall survival; PBSC: Peripheral blood stem cell; PRF-AML: Primary refractory acute myeloid leukemia; RI: Relapse incidence; RIC: Reduced intensity conditioning regimen; UD: Unrelated donor

\section{Acknowledgements}

We thank all the European Group for Blood and Marrow Transplantation (EBMT) participating centers and national registries for providing patients to the study and data managers for their valuable contribution (Additional file 1). Supplementary information is available at the EBMT Web site.

\section{Funding}

Not applicable.

\section{Availability of data and materials}

$E B, M L, M M$, and AN had full access to all the data in the study (available upon data specific request).

\section{Authors' contributions}

$E B, M L, M M$, and $A N$ designed the study or analyzed the data (or both). MS, $G E, R S, J F, H K, A G, K S, A Z, D B, S M, W B, N M, P K, I B, N K, A V, M G, E H, E H, J E$, and MM provided important clinical data. All authors contributed to the writing of the report and approved the final version of the article.

\section{Competing interests}

The authors declare that they have no competing interests.

\section{Consent for publication}

Not applicable.

\section{Ethics approval and consent to participate}

The study protocol was approved by the institutional review board at each site and complied with country-specific regulatory requirements. The study was conducted in accordance with the Declaration of Helsinki and Good Clinical Practice guidelines. Patients provide informed consent authorizing the use of their personal information for research purposes.

\section{Publisher's Note}

Springer Nature remains neutral with regard to jurisdictional claims in published maps and institutional affiliations.

\footnotetext{
Author details

'Service d'Hématologie Clinique et de Thérapie Cellulaire, Hôpital Saint Antoine, APHP, 184 rue du faubourg Saint-Antoine, 75571 Paris, Cedex 12, France. ${ }^{2}$ Acute Leukemia Working Party Office, Hôpital Saint Antoine, APHP, Paris, France. ${ }^{3}$ Department of Medicine A/Hematology and Oncology, University of Muenster, Muenster, Germany. ${ }^{4}$ Medizinische Klinik und Poliklinik I, Universitätsklinikum, Dresden, Germany. ${ }^{5}$ Deutsche Klinik für Diagnostik, KMY, Zentrum, Wiesbaden, Germany. ${ }^{6}$ Faculty of Medicine and Medical Center, Hematology, Oncology and Stem Cell Transplantation, University of Freiburg, Freiburg im Breisgau, Germany. ${ }^{7}$ Klinikum Grosshadern, Med. Klinik III, München, Germany. ${ }^{8}$ Department of Hematology, Hemostasis, Oncology and Stem Cell Transplantation, Hannover Medical School, Hannover, Germany. ${ }^{9}$ Medizische Klinik, Paracelsus Medizinische Privatuniversität, Nürnberg, Germany. ${ }^{10}$ Bone Marrow Transplantation Center, University Hospital Eppendorf, Hamburg, Germany. ${ }^{11}$ Klinik fuer Innere Medizin III, Universtätklinikum, Ulm, Germany. ${ }^{12}$ Department of Internal Medicine II, Würzburg University Medical Center, Würzburg, Germany. ${ }^{13}$ Medical Department, Hematology and Oncology, University of Tuebingen, Tübingen, Germany. ${ }^{14}$ Hematology, CHU de Bordeaux, Bordeaux, France. ${ }^{15}$ Department of Internal Medicine I, Bone Marrow Transplantation Unit, Medical University of Vienna, Vienna, Austria. ${ }^{16}$ Charite-Campus Benjamin
}

Franklin Universitaetsmedizin Berlin Klinik III- Hematologie u Onkologie, Hindenburgdamm, Berlin, Germany. ${ }^{17}$ Department of Stem Cell Transplantation, University Medical Center Hamburg-Eppendorf, Hamburg, Germany. ${ }^{18}$ Department of Clinical Hematology, Institute of Hematology and Blood Transfusion, Prague, Czech Republic. ${ }^{19}$ Division of Stem Cell Transplantation and Immunotherapy, University of Kiel, Kiel, Germany. ${ }^{20}$ Department of Haematology/Oncology, University Hospital Regensburg, Regensburg, Germany. ${ }^{21}$ Medizinische Klinik Klinikum, Augsburg, Germany. ${ }^{22}$ Hospital Clinic Institut d'investigacions Biomèdiques August Pi i Sunyer, Barcelona, Spain. ${ }^{23}$ Chaim Sheba Medical Center, Tel Hashomer, Israel.

Received: 1 May 2017 Accepted: 16 June 2017

Published online: 24 June 2017

\section{References}

1. Cheson BD, Bennett JM, Kopecky KJ, et al. Revised recommendations of the International Working Group for Diagnosis, Standardization of Response Criteria, Treatment Outcomes, and Reporting Standards for Therapeutic Trials in Acute Myeloid Leukemia. J Clin Oncol. 2003;21 (24):4642-9.

2. Slovak ML, Kopecky KJ, Cassileth PA, et al. Karyotypic analysis predicts outcome of preremission and postremission therapy in adult acute myeloid leukemia: a Southwest Oncology Group/Eastern Cooperative Oncology Group Study. Blood. 2000;96(13):4075-83.

3. Thol F, Schlenk RF, Heuser M, Ganser A. How I treat refractory and early relapsed acute myeloid leukemia. Blood. 2015;126(3):319-27.

4. Ferguson P, Hills RK, Grech A, et al. An operational definition of primary refractory acute myeloid leukemia allowing early identification of patients who may benefit from allogeneic stem cell transplantation. Haematologica. 2016;101(11):1351-8.

5. Revesz D, Chelghoum Y, Le QH, Elhamri M, Michallet M, Thomas X. Salvage by timed sequential chemotherapy in primary resistant acute myeloid leukemia: analysis of prognostic factors. Ann Hematol. 2003;82(11):684-90.

6. Duval M, Klein JP, He W, et al. Hematopoietic stem-cell transplantation for acute leukemia in relapse or primary induction failure. J Clin Oncol. 2010; 28(23):3730-8

7. Nagler A, Savani BN, Labopin M, et al. Outcomes after use of two standard ablative regimens in patients with refractory acute myeloid leukaemia: a retrospective, multicentre, registry analysis. Lancet Haematol. 2015;2(9):e384-92.

8. Gyurkocza B, Lazarus HM, Giralt S. Allogeneic hematopoietic cell transplantation in patients with AML not achieving remission: potentially curative therapy. Bone Marrow Transplant. 2017. doi:10.1038/bmt.2017.8 [Epub ahead of print].

9. Fung HC, Stein A, Slovak M, et al. A long-term follow-up report on allogeneic stem cell transplantation for patients with primary refractory acute myelogenous leukemia: impact of cytogenetic characteristics on transplantation outcome. Biol Blood Marrow Transplant. 2003;9(12):766-71.

10. Michallet $\mathrm{M}$, Thomas $\mathrm{X}$, Vernant JP, et al. Long-term outcome after allogeneic hematopoietic stem cell transplantation for advanced stage acute myeloblastic leukemia: a retrospective study of 379 patients reported to the Societe Francaise de Greffe de Moelle (SFGM). Bone Marrow Transplant. 2000;26(11):1157-63.

11. Wattad M, Weber D, Dohner $K$, et al. Impact of salvage regimens on response and overall survival in acute myeloid leukemia with induction failure. Leukemia. 2017;31 (6):1306-13. doi:10.1038/leu.2017.23 Epub 2017 Jan 19.

12. Craddock C, Labopin M, Pillai S, et al. Factors predicting outcome after unrelated donor stem cell transplantation in primary refractory acute myeloid leukaemia. Leukemia. 2011;25(5):808-13.

13. Jabbour E, Daver N, Champlin R, et al. Allogeneic stem cell transplantation as initial salvage for patients with acute myeloid leukemia refractory to high-dose cytarabine-based induction chemotherapy. Am J Hematol. 2014:89(4):395-8.

14. Weisdorf DJ. How closely related is graft-vs-leukemia to donor/recipient disparity? Best Pract Res Clin Haematol. 2010;23(4):525-8.

15. de Lima M, Champlin RE, Thall PF, et al. Phase I/II study of gemtuzumab ozogamicin added to fludarabine, melphalan and allogeneic hematopoietic stem cell transplantation for high-risk CD33 positive myeloid leukemias and myelodysplastic syndrome. Leukemia. 2008;22(2):258-64.

16. Sorror ML, Sandmaier BM, Storer BE, et al. Comorbidity and disease status based risk stratification of outcomes among patients with acute myeloid leukemia or myelodysplasia receiving allogeneic hematopoietic cell transplantation. J Clin Oncol. 2007;25(27):4246-54. 
17. Lim Z, Brand R, Martino R, et al. Allogeneic hematopoietic stem-cell transplantation for patients 50 years or older with myelodysplastic syndromes or secondary acute myeloid leukemia. J Clin Oncol. 2010;28(3):405-11.

18. Woolfrey A, Lee SJ, Gooley TA, et al. HLA-allele matched unrelated donors compared to HLA-matched sibling donors: role of cell source and disease risk category. Biol Blood Marrow Transplant. 2010;16(10):1382-7.

19. Schlenk RF, Dohner K, Mack S, et al. Prospective evaluation of allogeneic hematopoietic stem-cell transplantation from matched related and matched unrelated donors in younger adults with high-risk acute myeloid leukemia: German-Austrian trial AMLHD98A. J Clin Oncol. 2010;28(30):4642-8.

20. Ringden O, Pavletic SZ, Anasetti C, et al. The graft-versus-leukemia effect using matched unrelated donors is not superior to $\mathrm{HLA}$-identical siblings for hematopoietic stem cell transplantation. Blood. 2009;113(13):3110-8.

21. Saber W, Opie S, Rizzo JD, Zhang MJ, Horowitz MM, Schriber J. Outcomes after matched unrelated donor versus identical sibling hematopoietic cell transplantation in adults with acute myelogenous leukemia. Blood. 2012; 119(17):3908-16.

22. Gooley TA, Chien JW, Pergam SA, et al. Reduced mortality after allogeneic hematopoietic-cell transplantation. N Engl J Med. 2010;363(22):2091-101.

23. Lee $\mathrm{SJ}$, Klein J, Haagenson $M$, et al. High-resolution donor-recipient HLA matching contributes to the success of unrelated donor marrow transplantation. Blood. 2007;110(13):4576-83.

24. Karanes C, Nelson GO, Chitphakdithai P, et al. Twenty years of unrelated donor hematopoietic cell transplantation for adult recipients facilitated by the National Marrow Donor Program. Biol Blood Marrow Transplant. 2008; 14(9 Suppl):8-15.

25. Malard F, Chevallier P, Guillaume T, et al. Continuous reduced nonrelapse mortality after allogeneic hematopoietic stem cell transplantation: a singleinstitution's three decade experience. Biol Blood Marrow Transplant. 2014; 20(8):1217-23.

26. Bacigalupo A, Ballen $K$, Rizzo D, et al. Defining the intensity of conditioning regimens: working definitions. Biol Blood Marrow Transplant. 2009;15(12):1628-33.

27. Schmid C, Schleuning M, Ledderose G, Tischer J, Kolb HJ. Sequential regimen of chemotherapy, reduced-intensity conditioning for allogeneic stem-cell transplantation, and prophylactic donor lymphocyte transfusion in high-risk acute myeloid leukemia and myelodysplastic syndrome. J Clin Oncol. 2005;23(24):5675-87.

28. Glucksberg H, Storb R, Fefer A, et al. Clinical manifestations of graft-versushost disease in human recipients of marrow from $\mathrm{HL}$-A-matched sibling donors. Transplantation. 1974;18(4):295-304.

29. Terwey TH, Vega-Ruiz A, Hemmati PG, et al. NIH-defined graft-versus-host disease after reduced intensity or myeloablative conditioning in patients with acute myeloid leukemia. Leukemia. 2012;26(3):536-42.

30. Dohner H, Estey EH, Amadori S, et al. Diagnosis and management of acute myeloid leukemia in adults: recommendations from an international expert panel, on behalf of the European LeukemiaNet. Blood. 2010;115(3):453-74.

31. Arora M, Weisdorf DJ, Spellman SR, et al. HLA-identical sibling compared with 8/8 matched and mismatched unrelated donor bone marrow transplant for chronic phase chronic myeloid leukemia. J Clin Oncol. 2009;27(10):1644-52.

32. Ringden $\mathrm{O}$, Labopin $\mathrm{M}$, Ciceri $\mathrm{F}$, et al. Is there a stronger graft-versus-leukemia effect using HLA-haploidentical donors compared with HLA-identical siblings? Leukemia. 2016;30(2):447-55.

33. Barrett AJ, Battiwalla M. Relapse after allogeneic stem cell transplantation. Expert Rev Hematol. 2010;3(4):429-41.

34. Raanani P, Dazzi F, Sohal J, et al. The rate and kinetics of molecular response to donor leucocyte transfusions in chronic myeloid leukaemia patients treated for relapse after allogeneic bone marrow transplantation. $\mathrm{Br} J$ Haematol. 1997;99(4):945-50.

35. Schmid C, Schleuning M, Schwerdtfeger R, et al. Long-term survival in refractory acute myeloid leukemia after sequential treatment with chemotherapy and reduced-intensity conditioning for allogeneic stem cell transplantation. Blood. 2006;108(3):1092-9.

36. Stelljes M, Bornhauser M, Kroger M, et al. Conditioning with 8-Gy total body irradiation and fludarabine for allogeneic hematopoietic stem cell transplantation in acute myeloid leukemia. Blood. 2005;106(9):3314-21.

37. Byrd JC, Mrozek K, Dodge RK, et al. Pretreatment cytogenetic abnormalities are predictive of induction success, cumulative incidence of relapse, and overall survival in adult patients with de novo acute myeloid leukemia: results from Cancer and Leukemia Group B (CALGB 8461). Blood. 2002; 100(13):4325-36
38. Grimwade D, Hills RK, Moorman AV, et al. Refinement of cytogenetic classification in acute myeloid leukemia: determination of prognostic significance of rare recurring chromosomal abnormalities among 5876 younger adult patients treated in the United Kingdom Medical Research Council trials. Blood. 2010;116(3):354-65.

39. Tsirigotis $P$, Byrne M, Schmid C, et al. Relapse of AML after hematopoietic stem cell transplantation: methods of monitoring and preventive strategies. A review from the ALWP of the EBMT. Bone Marrow Transplant. 2016;51(11): $1431-8$.

40. Sorror M, Storer B, Sandmaier BM, et al. Hematopoietic cell transplantationcomorbidity index and Karnofsky performance status are independent predictors of morbidity and mortality after allogeneic nonmyeloablative hematopoietic cell transplantation. Cancer. 2008;112(9):1992-2001.

41. Guilfoyle R, Demers A, Bredeson C, et al. Performance status, but not the hematopoietic cell transplantation comorbidity index ( $\mathrm{HCT}-\mathrm{Cl})$, predicts mortality at a Canadian transplant center. Bone Marrow Transplant. 2009; 43(2):133-9.

42. Craddock C, Jilani N, Siddique $\mathrm{S}$, et al. Tolerability and clinical activity of post-transplantation azacitidine in patients allografted for acute myeloid leukemia treated on the RICAZA trial. Biol Blood Marrow Transplant. 2016; 22(2):385-90.

43. de Lima M, Giralt S, Thall PF, et al. Maintenance therapy with low-dose azacitidine after allogeneic hematopoietic stem cell transplantation for recurrent acute myelogenous leukemia or myelodysplastic syndrome: a dose and schedule finding study. Cancer. 2010;116(23):5420-31.

44. Oshikawa G, Kakihana K, Saito M, et al. Post-transplant maintenance therapy with azacitidine and gemtuzumab ozogamicin for high-risk acute myeloid leukaemia. Br J Haematol. 2015;169(5):756-9.

45. Pusic I, Choi J, Fiala MA, et al. Maintenance therapy with decitabine after allogeneic stem cell transplantation for acute myelogenous leukemia and myelodysplastic syndrome. Biol Blood Marrow Transplant. 2015;21(10):1761-9.

46. Craddock C, Labopin M, Robin M, et al. Clinical activity of azacitidine in patients who relapse after allogeneic stem cell transplantation for acute myeloid leukemia. Haematologica. 2016;101(7):879-83.

47. Bug G, Burchert A, Wagner EM. Phase I/II study of the deacetylase inhibitor panobinostat as maintenance therapy after an allogeneic stem cell transplantation in patients with high-risk MDS or AML: the Panobest-trial. Blood Abstract. 2015;126:4344.

48. Battipaglia G, Ruggeri A, Massoud R, et al. Efficacy and feasibility of sorafenib as a maintenance agent after allogeneic hematopoietic stem cell transplantation for Fms-like tyrosine kinase 3-mutated acute myeloid leukemia. Cancer. 2017. doi:10.1002/cncr.30680 [Epub ahead of print].

\section{Submit your next manuscript to BioMed Central and we will help you at every step:}

- We accept pre-submission inquiries

- Our selector tool helps you to find the most relevant journal

- We provide round the clock customer support

- Convenient online submission

- Thorough peer review

- Inclusion in PubMed and all major indexing services

- Maximum visibility for your research

Submit your manuscript at www.biomedcentral.com/submit
Biomed Central 